\title{
Efficacy of an enterovaccine in recurrent episodes of diarrhea in the dog: a pilot study
}

\author{
Matteo Cerquetella ${ }^{1}$, Fulvio Laus ${ }^{1}$, Fabiana Speranzini², Cristina Carnevali ${ }^{3}$, \\ Andrea Spaterna ${ }^{1}$, Edda Battaglia ${ }^{4}$ and Gabrio Bassotti ${ }^{5}$ \\ ${ }^{1}$ School of Medical Veterinary Sciences. University of Camerino. Matelica, Italy. ${ }^{2}$ DVM, Private Practitioner. \\ Genga, Italy. ${ }^{3}$ DVM, Private Practitioner. Montefano, Italy. ${ }^{4}$ Gastroenterology Section. Cardinal Massaja Hospital. Asti, \\ Italy. ${ }^{5}$ Gastroenterology and Hepatology Section. Department of Clinical and Experimental Medicine. Santa Maria della \\ Misericordia Hospital. Perugia, Italy
}

\begin{abstract}
Recurrent episodes of self-limiting diarrhea in the dog, due to sudden dietary changes and to stressful or exciting situations, are conditions sometimes difficult to treat. Colifagina ${ }^{\circledast}$, a commercially available bacterial enterovaccine, showed, in previous studies performed on experimentally induced colitis in mice, to be able to improve both disease activity index and histological appearance, increase colonic secretion of IgA, and reduce inflammatory chemokine secretion. In the present study Colifagina ${ }^{\circledR}$ was administered to five dogs presenting recurrent episodes of self-limiting diarrhea and to one dog presenting chronic diarrhea. During the follow-up period, almost all patients decreased the number of episodes of abnormal defecation and the fecal score of such episodes improved in five out of six dogs. Even if further studies are needed to understand the exact potential of the compound, in dogs presenting recurrent episodes of self-limiting diarrhea due to sudden dietary changes and/or stressing or exciting situations, Colifagina ${ }^{\circledR}$ seems to be helpful in managing most of these patients.
\end{abstract}

Key words: Diarrhea. Dogs. Enterovaccine. Treatment.

Cerquetella M, Laus F, Speranzini F, Carnevali C, Spaterna A, Battaglia E, Bassotti G. Efficacy of an enterovaccine in recurrent episodes of diarrhea in the dog: a pilot study. Rev Esp Enferm Dig 2012; 104: 65-68.

\section{INTRODUCTION}

In the dog, recurrent episodes of diarrhea may be due to repeated acute pathologic conditions, as well as to periodic exacerbation of chronic diseases, involving primarily or

Received: 03-10-11.

Accepted: 13-10-11

Correspondence: Matteo Cerquetella, School of Veterinary Medical Science, University of Camerino, Via Circonvallazione, 93/95, 62024 - Matelica (Macerata), Italy.

E-mail: matteo.cerquetella@unicam.it secondarily the gut. Among the causes that could justify the onset of diarrhea may be included sudden dietary changes in predisposed/particularly sensitive patients, and stressing or exciting situations in easily excitable patients or working dogs (1-4). Although such episodes are generally self-limiting, the patient often necessitates pharmacological and/or environmental management over time, the latter aimed at eliminating trigger causes (1); however, the first therapeutic approach is not always successful, while the second one is not easily applicable in all situations (3). Colifagina $^{\circledast}$, a bacterial enterovaccine, has been used in human medicine to treat intestinal symptoms in patients with colonic diverticulitis (5) and functional bloating (6). It contains deactivated whole bacteria and lysates of Escherichia coli 01, 02, 055, 0111, Bacillus pumilus, Morganella morgani, Alcaligenes faecalis, Shigella flexneri, Bacillus subtilis, Enterococcus faecalis and Proteus vulgaris, and has been shown to improve experimental colitis in experimental animal models (7). This improvement was achieved by inducing immunoglobulins secretion and ameliorating both disease activity index and histological appearance, by means of an increased colonic secretion of IgA, and also a significant reduction of inflammatory cytokine and chemokine secretion (7).

Aim of the present study was to evaluate the possible clinical efficacy of orally administered Colifagin ${ }^{\circledast}$ in managing dogs presenting recurrent episodes of self-limiting diarrhea; to our knowledge, this is the first clinical study using that enterovaccine in dog's colitis.

\section{MATERIALS AND METHODS}

\section{Patients}

Five dogs presenting recurrent episodes of self-limiting diarrhea from two-six months, and one dog presenting chronic diarrhea unresponsive to treatment, were included in the study. The relevant data are reported in table I. 
Table I. Clinical history and findings related to defecation at T0 and during the follow-up period.

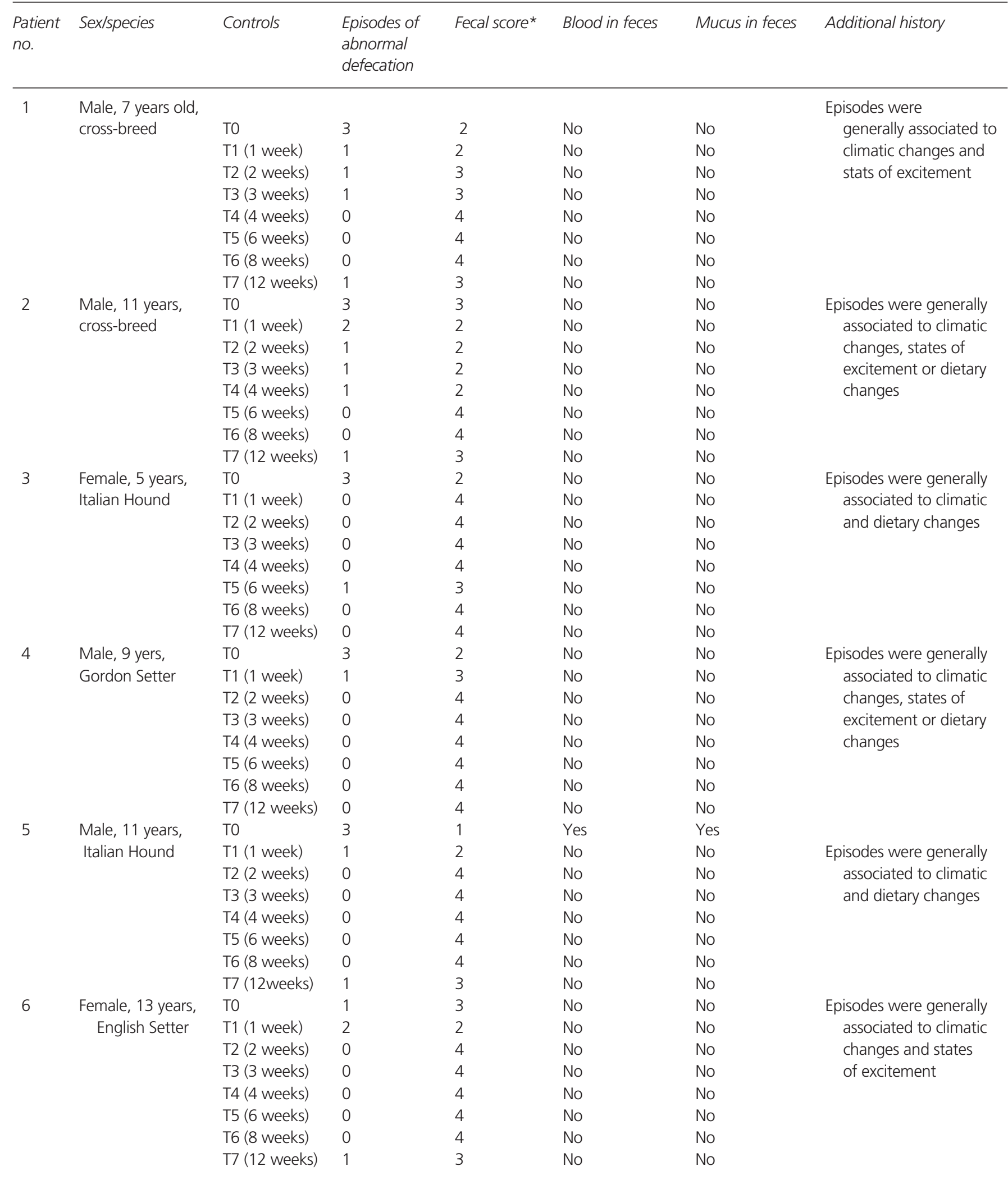

* Fecal scoring system: score 1 -watery stool; score 2 -soft non formed stool; score 3 -soft formed stool; score 4 -solid stool; score 5 -extremely dry stool (adapted from reference 3 ). 


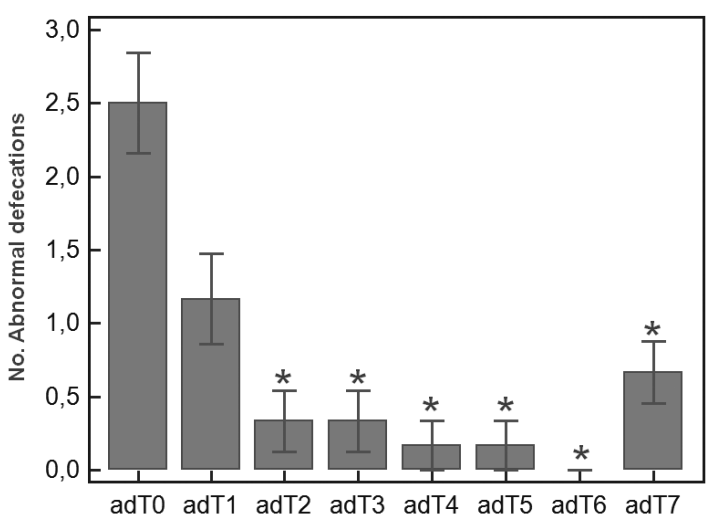

Fig. 1. Number of abnormal defecations during treatment and followup. ${ }^{*}=$ significantly different compared to TO.

Before study inclusion, for each patient history collection, clinical evaluation, complete hematological and biochemical evaluation (Haematology analyser, Cell-Dyn ${ }^{\circledR} 3500$, Abbott, USA and Automatic analyzer BT 3000 plus $^{\circledast}$, Biotecnica Instruments, Italy), as well as serial copro-microscopic examinations (3 consecutive days), including research for Giardia lamblia $\left(\mathrm{SNAP}^{\mathrm{TM}}\right.$ test GIARDIA, IDEXX Laboratories, USA), in order to exclude intestinal parasite and/or the other most important diseases causing diarrhea, were obtained. No obvious causes of diarrhea were thus found.

\section{Treatment protocol}

All patients were given orally, once a day for one month, an ampoule of Colifagina ${ }^{\oplus}$ (ABC FARMACEUTICI S.p.a., Torino, Italy) oral suspension, which contains deactivated whole bacteria and lysates of Escherichia coli 01, 02, 055,

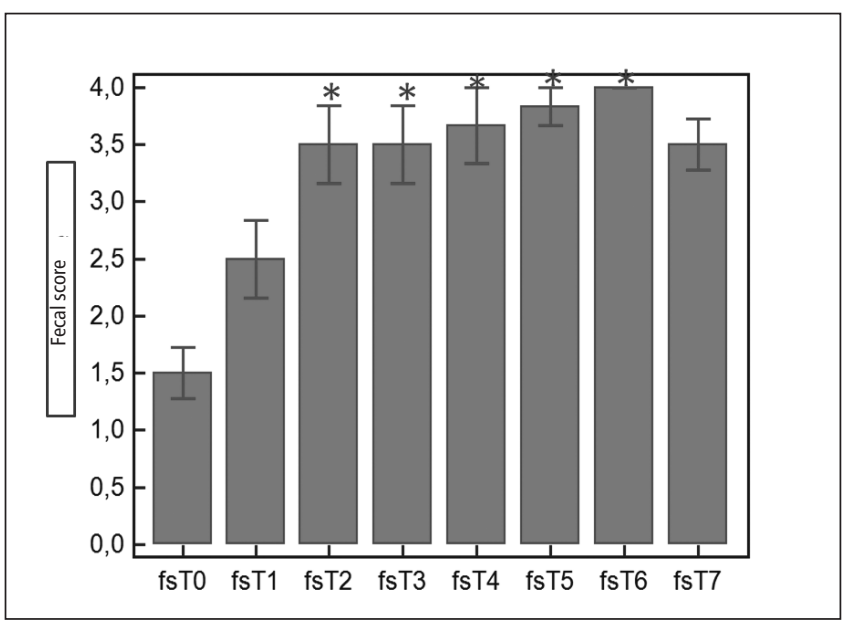

Fig. 2.Fecal score during treatment and follow-up. * = significantly different compared to TO.
0111 (80 billions), Bacillus pumilus (5 billions), Morganella morgani (4 billions), Alcaligenes faecalis (4 billions), Shigella flexneri (4 billions), Bacillus subtilis (1 billion), Enterococcus faecalis (1 billion) and Proteus vulgaris (1 billion).

Patients were evaluated clinically at the beginning of the study (T0), and then after 1 week (T1), 2 weeks (T2), 3 weeks (T3), 4 weeks (T4), 6 weeks (T5), 8 weeks (T6), and 12 weeks (T7). All patients underwent serial copro-microscopic and haematobiochemical evaluation, as previously reported, at T0, T4, T6 and T7. Tests for Giardia were also performed at T0, T4 and T7. Body weight was evaluated daily, while the weekly number of abnormal defecation gross appearance of stool was constantly monitored and any changes recorded, in accordance with a validated fecal scoring system (3). The scoring system was defined as: score 1 = watery stool; score 2 = soft non formed stool; score $3=$ soft formed stool; score 4 = solid stool; score 5 = extremely dry stool. The eventual presence of blood and/or mucus was also recorded, as well as the presence of side effects.

Patients 1 and 2 were followed clinically, including stool gross appearance monitoring, for a period longer than 3 months, T7 (respectively other 4 and other 9 months).

During the therapeutic trial and for the follow up period, it was not possible to modify dogs' general life conditions (environment, habits, etc.).

The study was approved by the Ethical Committee of University of Camerino.

\section{Statistical analysis}

Data were analyzed by means of analysis of variance (ANOVA) for repeated measures, and the single time interval compared by the Student's t-test (Bonferroni correction) for paired data.

\section{RESULTS}

The number of abnormal defecation episodes and the fecal score of each episode for all patients during the follow-up period are summarized in table I. Treatment with Colifagina $^{\otimes}$ markedly improved both the number of episodes of abnormal defecation (Fig. 1) and the fecal score (Fig. 2), starting from the first week of treatment. Interestingly, this significant improvement lasted up to 4 weeks after the end of treatment, and in the two dogs followed for longer periods ( 4 and 9 months), only the first had a single episode of abnormal defecation. No side effects and/or biochemical abnormalities were documented during the treatment and the follow-up.

\section{DISCUSSION}

This pilot study showed that Colifagina ${ }^{\circledR}$ is able to improve in a significant manner symptoms in dogs with 
recurrent episodes of diarrhea not due to obvious causes. The rationale of the present study finds its basis in the fact that in dogs a sudden dietary change could determine, in certain patients, phenomena of maldigestion and malabsorption that could lead in turn to bacterial overgrowth and diarrhea (2), and also to the fact that stress is considered as well a cause of diarrhea (1).

Thus, on the basis that it is considered likely that in determining colitis a reduction in protective mechanisms (e.g. lower IgA secretion, inefficacy of the mucosal barrier) could play an important role (1), and given that in experimental animal models of colitis Colifagina ${ }^{\circledR}$ showed the capacity of improving disease activity index and histological appearance, increasing colonic secretion of $\operatorname{IgA}$, and reducing inflammatory cytokine and chemokine secretion (7), we hypothesized a possible role for that bacterial lysate preparation in managing dogs with recurrent episodes of self-limiting diarrhea due to sudden dietary changes and/or stressing or exciting situations.

Of course, this study has limitations, in that we were unable (due to ethical restrictions) to provide a control group and to perform endoscopic examinations, useful to define the clinical picture $(8,9)$. However, the results we obtained should prompt further in depth research on this topic, by using larger number of subjects, placebo-controlled design, and endoscopic examinations.

In conclusion, oral treatment with Colifagina ${ }^{\circledR}$ is able to improve symptoms and fecal consistency in dogs with recurrent episodes of diarrhea not due to obvious causes, and this improvement lasts up to one month (and probably longer) after the end of treatment. Further studies are warranted to confirm these findings and provide more data on the possible value of this therapeutic approach.

\section{ACKNOWLEDGMENTS}

We are warmly indebted to Dr Alberto Giraudi, ABC Farmaceutici, Torino, Italy for the kind gift of the enterovaccine ampoules.

\section{REFERENCES}

1. Simpson JW. Diet and large intestinal disease in dogs and cats. J Nutr 1998;128:2717S-2722S

2. Willard MD. Malattie del tratto intestinale. In: Nelson RW, Couto CG, editors. Medicina interna del cane e del gatto. $3^{\text {rd }}$ ed. Milano, Italy: Elsevier Masson Srl; 2007. p. 441-75.

3. Westermarck E. Valutazione clinica di pazienti con diarrea cronica. In: Steiner JM, editor. Gastroenterologia del cane e dal gatto. Milano, Italia: Elsevier Srl; 2009. p. 118-9.

4. Stavisky J, Radford AD, Gaskell R, Dawson S, German A, Parsons B, et al. A case-control study of pathogen and lifestyle risk factors for diarrhoea in dogs. Prev Vet Med 2011;99: 185-92.

5. Dughera L, Serra AM, Battaglia E, Tibaudi D, Navino M, Emanuelli G. Acute recurrent diverticulitis is prevented by oral administration of a polybacterial lysate suspension. Minerva Gastroenterol Dietol 2004; 50:149-53.

6. Grassini M, Battaglia E, Verna C, Niola P, Repici A, Corazzi N, Bassotti $\mathrm{G}$. Improvement of functional bloating by an enterovaccine: a preliminary study. Rev Esp Enferm Dig 2009;101:619-22.

7. Vetrano S, Correale C, Borroni EM, Pagano N, Savino B, Locati M, Malesci A, Repici A, Danese S. Colifagina, a novel preparation of 8 lysed bacteria ameliorates experimental colitis. Intern J Immunopathol Pharmacol 2008;21:401-7.

8. Sherding RG. Diseases of the large intestine. In: Tams TR, editor. Handbook of small animal gastroenterology. $2^{\text {nd }}$ ed. St. Louis, USA: Saunders-Elsevier Science; 2003. p. 251-85.

9. Jergens AE, Zoran DL. Diseases of the colon and rectum. In: Hall EJ, Simpson JW, Williams DA, editors. BSAVA Manual of Canine and Feline Gastroenterology. $2^{\text {nd }}$ ed. Gloucester, England: British Small Animal Veterinary Association; 2005. p. 203-12. 